\title{
Reduction of the Clipping Noise for OFDM/OQAM System
}

\author{
Guobing Cheng, Qifu Lv, Shaoqian Li, Lisha Gong, Binhong Dong, Su Hu \\ National Key Laboratory of Science and Technology on Communications, \\ University of Electronic Science and Technology of China, Chengdu, China \\ Email: guobingcheng12@163.com
}

Received August, 2013

\begin{abstract}
Orthogonal frequency division multiplex/offset QAM (OFDM/OQAM) has been proven to be a promising multi-carrier modulation (MCM) technique for the transmission of signals over multipath fading channels. However, OFDM/OQAM has also the intrinsic disadvantage of high peak-to-average-power ratio (PAPR) that should be alleviated. This paper focuses on the reduction of the clipping noise and out-of band radiation caused by the clipping process. The basic principle is to estimate the clipping noise and then eliminate it from the received signal. Analysis and simulation results show that, with one time iteration, the proposed method can effectively improve the bit error ratio (BER) performance.
\end{abstract}

Keywords: OFDM/OQAM; PAPR Reduction; Clipping Noise

\section{Introduction}

As well as the other kinds of MCM systems, since the resulting OFDM/OQAM signal is the summation over all the statistically independent subcarriers, it also has the intrinsic characteristic of high peak-to-ratio (PAPR). And for a given power amplifier, it always has a certain linear amplification range that distortion will be created when working at the nonlinear range. Furthermore, the power amplification of signals having a large dynamic range may introduce inter-modulation between subcarriers and cause interferences [1]. These distortion and interference lead to performance degradation, which is in close relation with the PAPR of the signal.

There have been some literatures attributed to the reduction of the PAPR in OFDM/OQAM system. In [2], the authors derived an approximate expression of the well-known complementary cumulative density function (CCDF) for OFDM/OQAM system. It concluded that the expression of CCDF of OFDM/OQAM is similar to that of the OFDM system and the common orthogonal pulse shapes also can provide optimal CCDF performance. In [3], the authors analyzed the application of the partial transmit sequence (PTS) method to OFDM/OQAM system and a novel algorithm based on dynamic programming joint optimization has been presented to reduced the PAPR. Corresponding to the selective mapping (SLM) method in OFDM system [4], the authors in [5] proposed an overlapped selective mapping (OSLM) method for OFDM/OQAM system. However, a pulse shape that may cover several OFDM symbols is introduced in OFDM/ OQAM system, resulting in the typical SLM and PTS
PAPR reduction methods of traditional OFDM system cannot be directly applied to the OFDM/OQAM system. And this disadvantage can be only partly overcome at the price of higher complexity and/or poorer system performance [4-7].

On the other hand, clipping is a simple and efficient method for reduction of PAPR that there have been many literatures for that of the traditional OFDM system. However, there has few public literature that been attributed the clipping method to OFDM/OQAM system. In this paper, we consider the application of clipping method to OFDM/OQAM system and focus on the reduction of the clipping noise. Firstly, we deduce the expression of the clipped OFDM/OQAM signal. Then a novel clipping noise reduction algorithm of traditional OFDM system proposed in [8] is introduced and we analyze the applying of this algorithm to the OFDM/ OQAM system. The analysis and simulation results show that the iterative clipping noise reduction method of the OFDM system can be directly applied to the OFDM/ OQAM system and it can efficiently improve the system performance. Furthermore, the out-of-band radiation caused by clipping process with and without filter is discussed in this paper.

\section{OFDM/OQAM System Model and PAPR Definition}

\subsection{System Model}

The baseband version of a continuous-time OFDM/ OQAM transmitted signal can be written as [9] 


$$
s(t)=\sum_{n=-\infty}^{+\infty} \sum_{m=0}^{M-1} a_{m, n} e^{j \phi_{m, n}} e^{j 2 \pi m v_{0} t} g\left(t-n \tau_{0}\right),
$$

with $M$ an even number of sub-carriers, $a_{m, n}$ the realvalued symbol conveyed by the sub-carrier of index $m$ during the symbol time of index $n, g$ the pulse shape, $v_{0}$ the subcarrier spacing and $\tau_{0}$ the time offset between the adjacent real part and imaginary part of an OFDM/ OQAM symbol. $v_{0}=1 / T_{0}=1 / 2 \tau_{0}$, with $T_{0}$ the duration of the complex-valued symbols. $\phi_{m, n}$ is an additional phase term given by

$$
\phi_{m, n}=\phi_{0}+\frac{\pi}{2}(m+n) \quad(\bmod \pi),
$$

where $\phi_{0}$ can be arbitrarily chosen.

For a distortion-free channel, perfect reconstruction is obtained owing to the following real orthogonal condition

$$
\mathfrak{R}\left\{\left\langle g_{m, n} \mid g_{p, q}\right\rangle\right\}=\mathfrak{R}\left\{\int g_{m, n}(t) g_{p, q}^{*}(t)\right\}=\delta_{m, p} \delta_{n, q}
$$

where $\mathfrak{R}\{\bullet\}$ is the taking real part operator. $\delta_{m, p}=1$ if $m=p$ and $\delta_{m, p}=0$ if $m \neq p$.

It is shown in equation (1) that the transmitted signal $s(t)$ is the summation over all the statistically independent subcarriers. If the number of subcarriers $M$ is large enough, the amplitude of $s(t)$ also varies in a large range, leading to high PAPR.

\subsection{PAPR Definition of OFDM/OQAM System}

The PAPR is an important parameter to measure the sensitivity to non-linear amplification of a transmission scheme having a non-constant envelope [5]. And the PAPR of the OFDM signals with $M$ carriers in discretetime version is defined as [4]

$$
\operatorname{PAPR}(d B)=10 \log _{10} \frac{\max _{k \in\{0, \ldots, M-1\}}\left\{\left|s_{k}\right|^{2}\right\}}{E\left\{\left|s_{k}\right|^{2}\right\}},
$$

where $s_{k}$ is the OFDM signal and $E\{*\}$ is the mean of $\{*\}$.

Since OFDM and OFDM/OQAM systems transmit the equivalent of one complex symbol at rate $1 / T_{0}$, it is reasonable to use equation (4) for PAPR measurement in OFDM/OQAM system [5].

The PAPR of the OFDM/OQAM system is also a random variable and its behavior is to compute the probability to exceed a given threshold $\gamma(d B)$ and the CCDF gives this probability for every $\gamma$, which is given by

$$
\mathrm{CCDF}=\mathrm{P}\{\mathrm{PAPR}>\gamma\} \text {. }
$$

Even though a pulse shape is introduced in OFDM/ OQAM system, it has been proven that the whole class of orthogonal prototypes such as the square root of raised cosine (SRRC) and isotropic orthogonal transform algorithm(IOTA), that are only nearly-orthogonal, also provide optimal CCDFs [2].

\section{Reduction of Clipping Noise in OFDM/OQAM System}

\subsection{Reduction of Clipping Noise in OFDM System}

A novel iterative estimation and cancellation of clipping noise algorithm for OFDM system has been proposed in [8]. It concluded that for an 802.11a system, the PAPR can be reduced to $4 \mathrm{~dB}$ while the system performance can be restored to less than $1 \mathrm{~dB}$ of the non-clipped case with only moderate complexity increase and with no bandwidth expansion. The detail principle of iterative clipping noise reduction is given in section II of [8].

\subsection{Reduction of the Clipping Noise in OFDM/OQAM System}

Now we apply the clipping noise reduction algorithm to the OFDM/OQAM system. Assume that the OFDM/ OQAM transmitted signal $s(t)$ is oversampled at time intervals $\Delta t=T_{0} / J M$, where $J$ is the oversampling factor. Then the resulting discrete version of $s(t)$ can be expressed as

$$
s_{n}=s(n \Delta t), n \in \mathbb{Z}
$$

Let $S_{n}$ pass through a clipper with a given threshold $A$, and then we get the clipped signal, denoted by $\bar{s}_{n}$ that

$$
\bar{S}_{n}=\left\{\begin{array}{ll}
S_{n} & \left|S_{n}\right| \leq A \\
A & \left|S_{n}\right|>A
\end{array} .\right.
$$

Because $s_{n}$ is the summation over a large number independent subcarriers, according to the law of large numbers, it can be characterized as a discrete complex stationary Gaussian process. Suppose that $s_{n}$ is routed to a device with memoryless nonlinearity. Applying the Bussgang's theorem [11], the output can be written as [10]

$$
\bar{s}_{n}=\alpha s_{n}+d_{n},
$$

where the distortion term $d_{n}$ is uncorrelated with $S_{n}$ and the attenuation factor $\alpha$ is a function of the clipping ratio $\gamma$, defined as $\gamma=A / P_{i n}$, with $P_{i n}$ the average power before clipping, that

$$
\alpha=1-e^{-\gamma^{2}}-\frac{\sqrt{\pi} \gamma}{2} \operatorname{erfc}(\gamma) \text {. }
$$

The block diagram of baseband equivalent transmitter and receiver with iterative clipping noise cancellation are given in Figure 1. 
Using (8), the term $\bar{C}$ in Figure 1 can be expressed as

$$
\bar{C}_{k}=\alpha C_{k}+D_{k} \quad \mathrm{k}=0, \ldots, \mathrm{JM}-1,
$$

where $C_{k}$ and $D_{k}$ are the DFT of $s_{n}$ and $d_{n}$ respectively.

Assuming perfect synchronization and following DFT, the signal at the receiver can be expressed as

$$
\begin{aligned}
Y_{k} & =H_{k} \bar{C}_{k}+Z_{k} \\
& =H_{k}\left(\alpha C_{k}+D_{K}\right)+Z_{k} \\
& =\alpha H_{k} C_{k}+Z_{k}+H_{k} D_{k}
\end{aligned}
$$

where $k=0, \ldots, J M-1, \quad H_{k}$ is the complex channel gain of the $k$-th sub-carrier that assumed to be perfectly known and $Z_{k}$ is the additive white Gaussian noise. It is shown in Equation 11 that the term $H_{k} D_{k}$ is the component caused by clipping process. Since the clipping course is known by the receiver side, estimation of $D_{k}$, denoted by $\hat{D}_{k}$, can be gotten by passing $\hat{C}_{k}$ through the same clipping and filtering process as in the transmitter, and then subtracted by $\alpha \hat{C}_{k}$ that

$$
\hat{D}_{k}=G_{k}-\alpha \hat{C}_{k}=\left(\alpha \hat{C}_{k}+\hat{D}_{k}\right)-\alpha \hat{C}_{k}=\hat{D}_{k}
$$

Then $\hat{D}_{k}$ is passed through the channel $H_{k}$ and we get that

$$
\begin{aligned}
\hat{Y}_{k} & =Y_{k}-H_{k} \hat{D}_{k} \\
& =\alpha H_{k} C_{k}+Z_{k}+H_{k}\left(D_{k}-\hat{D}_{k}\right)
\end{aligned}
$$

If $\hat{D}_{k}=D_{k}$, the clipping noise component in Equation 13 can be removed absolutely. On the other hand, from Equation 12, since the estimation of $D_{k}$ has little to do with the accuracy of $\hat{C}_{k}$, incremental gains diminish after the first iteration.

Furthermore, in order to remove the out-of-band radiation caused by clipping process, a pair of time-frequency domain transform has to be included both in transmitter and in each iteration process of receiver, as shown in Figure 1. This results in the increasing of calculation complexity and bit error ratio (BER). While in OFDM/ OQAM system, the out-of-band radiation of the clipped signal is still in the acceptable range. Therefore we can omit the out-of-band removal stage in both transmitter and receiver, as shown in the simulation results.

\section{Simulation Results}

In this section, it aims to evaluate the efficiency of the clipping noise cancellation algorithm in OFDM/OQAM system.

Figure 2 presents the BER to the signal-to-noise ratio (SNR) in AWGN channel for clipping noise removal with threshold $=6 \mathrm{~dB}$. It shows that, through clipping noise removal process, the system performance can be largely recovered. Secondly the results of 1 and 2 times iteration are almost overlapped, implying that 1 time iteration is enough, which is consistent with the analysis results.

In Figure 3, it compares the power spectrum density (PSD) for different threshold and with or without filter. It is shown that the out-of-band radiation is regrown by clipping. The traditional out-of-band removal method, i.e., padding zeros with the middle $(J-1) M$ points,

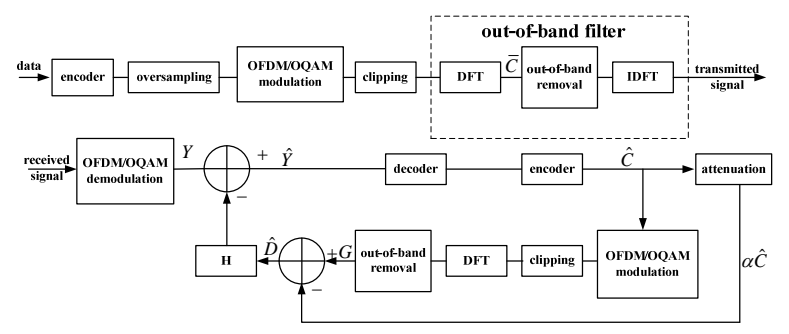

Figure 1. The baseband equivalent transmitter and receiver with iterative clipping noise cancellation.

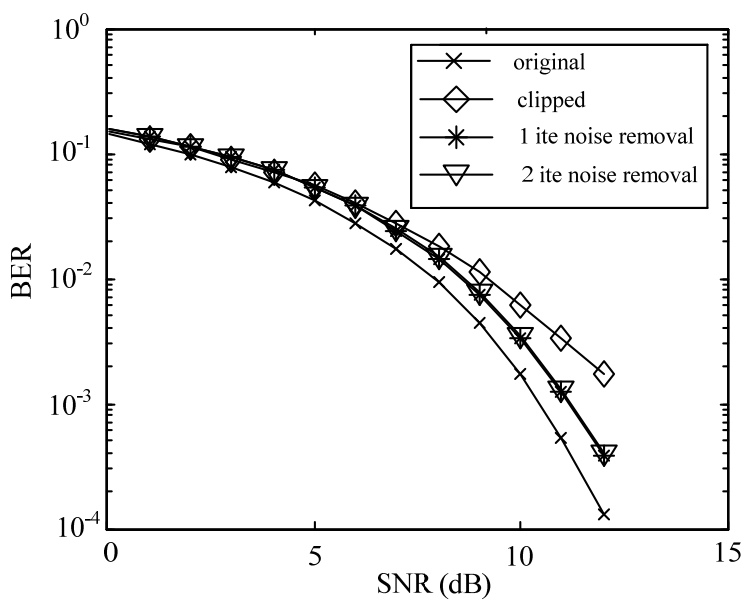

Figure 2. The BER to the SNR in AWGN channel for clipping noise removal with threshold $=6 \mathrm{~dB}$.

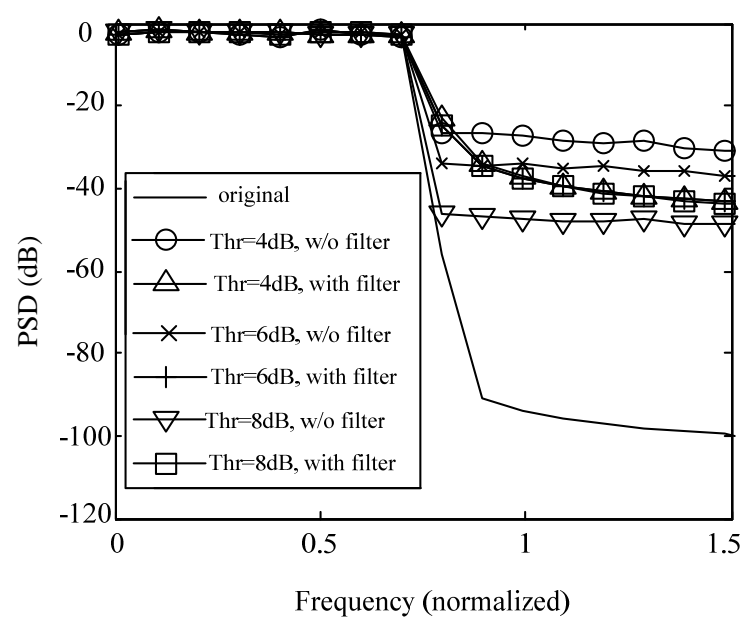

Figure 3. The PSD for OFDM/OQAM clipped signal with and without filter. 
has little effect on the out-of-band removal and it even becomes worse when threshold is high, e.g., threshold = $8 \mathrm{~dB}$ in Figure 3. On the other hand, since the clipping noise removal process includes the same process in transmitter, the including out-of-band removal will largely increase the computation complexity.

\section{Conclusions}

The reduction of clipping noise in OFDM/OQAM system is discussed in this paper. It is shown that the system performance can be largely recovered through iterative clipping noise cancellation. Furthermore, since the outof-band radiation of the clipped signal is at a considerable low level, there is no necessary to include the outof-band filtering process in OFDM/OQAM system.

\section{Acknowledgements}

This work is supported in part by the National Science Foundation of China under Grant number 61101101, National Grand Special Science and Technology Project of China under Grant No. 2010ZX03006-002-02, , Program for New Century Excellent Talents in University of China ((NCET110058), the Foundation Project of National Key Laboratory of Science and Technology on Communications under Grant 9140C020404120C0201, and Key Laboratory of Universal Wireless Communications, Beijing university of Posts and Telecommunications, Ministry of Education, China (No. KFKT2012102).

\section{REFERENCES}

[1] L. Hanzo, M. Miinster, B. J. Choi and T. Keller, “OFDM and MC-CDMA for Broadband Multi-Users Communications," WLANs and Broadcasting, IEEE press. Wiley Series, 2003.

[2] A. Skrzypczak, P. Siohan and J. P. Javaudin, “Analysis of the Peak-to-average Power Ratio for OFDM/ OQAM," IEEE 7th Workshop on Signal Processing Advances in Wireless Communications, July 2006.

[3] D. M. Qu, S. X. Lu and T. Jiang, "Multi-Block Joint Optimization for the Peak-to-average Power Ratio Reduction of FBMC-OQAM Signals,” IEEE Transactions on Signal Processing, Vol. 61, No. 7, 2013, pp. 1605-1613. doi:10.1109/TSP.2013.2239991

[4] R. W. Bauml, R. F. H. Fischer and J. B. Huber, "Reducing the Peak-to-Average Power Ratio of Multicarrier Modulation by Selected Mapping," Electronics Letters, Vol. 32, No. 22, 1996, pp. 2056-2057. doi:10.1049/el:19961384

[5] A. Skrzypczak, P. Siohan and J. P. Javaudin, "Reduction of the peak-to-average Power Ratio for OFDM-OQAM Modulation,” in Proc.63rd IEEE VTC, May 2006, pp. 2018-2022. doi:10.1109/LSP.2007.898351

[6] Y. Xiao, X. Lei, Q. Wen and S. Li, "A Class of Low Complexity PTS Techniques for PAPR Reduction in OFDM Systems,” IEEE, Signal Processing Letters, 2007. doi:10.1109/LSP.2007.898351

[7] Y. Xiao, S. Li, X. Lei and Y. Tang, "Clipping Noise Mitigation for Channel Estimation in OFDM Systems,” IEEE Communications Letters, 2006.

[8] H. Chen, A. M.Haimovich, "Iterative Estimation and Cancellation of Clipping Noise for OFDM Signals," IEEE Communication Letter, Vol. 7, No.7, 2003, pp. 305-307. doi:10.1109/LCOMM.2003.814720

[9] B. Le Floch, M. Alard and C. Berrou, "Coded Orthogonal Frequency Division Multiplex,” Proceedings of the IEEE, Vol. 83, 1995, pp. 982-996. doi:10.1109/5.387096

[10] H. Ochiai and H. Imai, "Performance Analysis of Deliberately Clipped OFDM Signals," IEEE Transactions Communications, Vol. 50, 2002, pp. 89-101. doi:10.1109/26.975762

[11] H. E. Rowe, "Memoryless Nonlinearities with Gaussian input: Elementary Results,” Bell System Technical Journal, Vol. 61, 1982, pp. 1519-1525. doi:10.1002/j.1538-7305.1982.tb04356.x 